\title{
Corrigendum: Pharmacoeconomic comparison of aripiprazole once-monthly and paliperidone palmitate from a head-to-head clinical trial in schizophrenia: a US analysis
}

\author{
Abstract \\ The authors wish to make the following corrections to their article: \\ Sapin C, Hartry A, Kamat SA, Beillat M, Baker RA, Eramo A. Pharmacoeconomic comparison of aripiprazole once-monthly and \\ paliperidone palmitate from a head-to-head clinical trial in schizophrenia: a US analysis. Drugs in Context 2016; 5: 212301. \\ DOI: 10.7573/dic.212301
}

\section{Corrigendum}

The authors regret that there is an error in the approach for deriving the bootstrapped $95 \%$ confidence intervals $(\mathrm{Cl})$, in reality these are all bootstrapped $90 \% \mathrm{Cl}$. Furthermore, precision of the statistical methods applied have been incorporated. The overall interpretation and conclusion of the results have not changed.

Deleted text is with strikethrough while new text is in italics and underlined.

\section{Abstract}

95\% Cl should be changed to $90 \% \mathrm{Cl}$.

\section{Assessments and statistical analyses}

Total cost outcomes (least squares mean per treatment group) were then estimated from an analysis of covariance model, including geographic region (Europe vs North Ameriea) and treatment as fixed effects as well as cost incurred during the 6 months before study entry and study drug exposure time as covariates. In a sensitivity analysis, adjustment for geographic region (Europe vs North America) was added.

90\% and $95 \%$ confidence intervals (Cls) for incremental effectiveness and cost were calculated using a bootstrapped, bias-corrected, accelerated nonparametric procedure with 10,000 iterations (a random seed of 1234 was used for deriving the 95\% Cls) [27].

As was done for the primary analysis of the QUALIFY study, Effectiveness outcomes (mean QLS and CGI-S score change from baseline) were analyzed using an analysis of covariance model similar to the one used for cost outcomes a mixed modet for repeated measures with an unstructured covariance matrix including baseline score-by-visit interaction, geographic region (Europe vs North America), visit, and treatment-by-visit interactions as fixed effects.

\section{Results}

Total costs were significantly lower for AOM 400 than PP ( $p=0.005$; Table 3). The cost of AOM 400 treatment during the QUALIFY study was approximately $9 \%$ lower $(p<0.001)$ than the cost of PP treatment, whereas the costs associated with services delivered by health-care professionals and inpatient or outpatient providers did not differ significantly between treatment groups (Table 3). Adjusting for geographic region did not change the results.

The mean $(950 \% \mathrm{Cl})$ change in QLS total score for patients treated with AOM 400 was $5.97(3.87 ; 8.08)$ compared with 2.85 $(0.56 ; 5.08)$ with PP. For effectiveness based on CGI-S, results also favored AOM over PP (Table 4), with mean $(95 \underline{0} \% \mathrm{Cl})$ change in CGI-S scores of $-0.59(-0.71 ;-0.47)$ with AOM 400 compared with $-0.37(-0.46 ;-0.27)$ with PP. Using a random seed of 1234 , the mean treatment difference (bootstrapped 95\% CI) was 3.15 (95\% Cl: -0.29; 6.88) and $-0.22(-0.05 ;-0.40)$ for QLS total score and CGI-S, respectively.

\section{Table 3}

Footnote: *Total cost outcomes were estimated from an analysis of covariance model, including geographic region (Europe vs North America) and treatment as fixed effects as well as cost incurred during the 6 months before study entry and study drug exposure time as covariates.

\section{Table 4}

$95 \% \mathrm{Cl}$ should be changed to $90 \% \mathrm{Cl}$. 